\title{
PRELIMINARY REPORT ON THE OCCURRENCE OF Chancelloria sp. IN THE ITAJAí BASIN, SOUTHERN BRAZIL
}

\section{PAULO SERGIO GOMES PAIM*, ITAMARIVO LEIPNITZ*, ANA LUCIA ZUCCATTI DA ROSA \& ATILA AUGUSTO STOCK DA ROSA}

\begin{abstract}
INTRODUCTION This note reports the first findings of fossil relicts in the sedimentary record of the Itajaí Basin (Santa Catarina State - Fig. 1). The fossil remains, which belong to Chancelloria sp., were recently presented to the $15^{\text {th }}$ Brazilian Paleontological Congress (Leipnitz et al. 1997) and support both a marine depositional environment and a Cambrian age of the uppermost unit of the Itajaí Group (Campo Alegre Formation). The conclusions about a Cambrian age and a marine environment are also supported by trace fossils recently described in the same succession (Netto \& Zucatti da Rosa 1997).
\end{abstract}

GEOLOGICAL SETTING The Itajaí Basin has been classically grouped with several other volcano-sedimentary basins of southern Brazil and interpreted as a late Precambrian to early Palaeozoic molasse basin of the Brasiliano Orogeny (Almeida 1967). Taking into account several geological evidence, the Camaqua (Rio Grande do Sul State) and Itajaí basins have been recently correlated to Pan-African basins (Vanrhynsdorp and Nama Basins) of southern Africa (Gresse et al 1996).

Until now, the only age constraint for the Itajaí Group was restricted to whole-rock $\mathrm{Rb} / \mathrm{Sr}$ isochron diagenetic/ sedimentation age of $581 \pm 48 \mathrm{Ma}$ (Macedo et al. 1984, Basei et al. 1987 ) and whole-rock $\mathrm{Rb} / \mathrm{Sr}$ isochron age of intrusive rocks $544 \pm 20 \mathrm{Ma}$ of the alkaline Apiuna Rhyelites and $546 \pm 10$ Ma of the Subida Granite (Basei et al. 1987). Therefore, the Itajaí Group would be older than $545 \mathrm{Ma}$, with a depositional age about $580 \mathrm{Ma}$, and hence ascribed to the Vendian Period.

Although a marine environment was previously suggested for the subaqueous facies of the Itajaí Group (e.g. Appi et al. 1987, Krebs et al. 1990, Mora 1993, Gresse et al. 1996), no conclusive evidence of a marine setting has been presented. Therefore, several previous papers use terms such as basinal or subaqueous sedimentation instead of marine (Basei et al. 1987, Appi \& Souza Cruz 1990, Rostirolla \& Figueira 1995). Furthermore, in other coeval Brazilian molasse basins, the only published evidence of marine life are represented by trace fossils described in the Camaqua Basin (Netto et al. 1992) and Camarinha Formation (Ciguel et al. 1992).

THE FOSSIL REMAINS The fossils were found approximately $2 \mathrm{~km}$ southeasterward from the main road of the region (BR 470) in a relatively small road cut located at $27^{\circ}$ $05^{\prime} 47^{\prime \prime} \mathrm{N}$ and $49^{\circ} 25^{\prime} 53^{\prime \prime} \mathrm{W}$ (Fig. 1) which is included within the lower part of the Campo Alegre Formation (Fig. 2). At least in this outcrop, the fossils occur within a single interval, about $0,5 \mathrm{~m}$ thick, (Figs. 3A and 3B), at the bedding planes of normal graded, thinly-bedded siltstones and claystones interpreted as the product of low-density turbidity currents (Tde) deposited in interchannel areas of a submarine-fan complex.

The fossil remains comprise amalgamated, sometimes broken sclerites presenting 4 to 6 tangential rays 2,0 to $7,5 \mathrm{~mm}$ long that thin out from the central 1 to $3 \mathrm{~mm}$ diameter disk (Fig. 3D). Some rays have a smooth, longitudinal depression on their axial portions (Fig. 3C). These remains were classified as follow:

Phylum and Class uncertain

Order Coeloscleritophora (Bengtson \& Missarzhevsky, 1981)

Family Chancelloriidae Walcott, 1920

Genus Chancelloria Walcott, 1920

FINAL REMARKS Chancelloria has been found in North America (Canada and USA), South America (Argentine), Europe (Spain, Great Britain, France, Italy and Greenland), Asia (Soviet Union, China and Iran), Africa (Senegal and Guinea) and Australia, as resumed in Table 1. The genus always occurs within Cambrian strata and hence is considered a Cambrian taxon (Morris 1992). Considering the worldwide occurrence of this fossil, mostly in Lower to Middle Cambrian strata, the assignement of the Campo Alegre Formation to the Cambrian Period is here proposed, in disagreement with the radiometric age of 580 Ma previously proposed for the Itajaí Group. In addition, a marine environment is now better supported for the stratigraphic level containing the fossil remains and hence a near or below sea level setting should be considered in future geological models proposed for the Itajaí Basin.

Acknowledgments To Conselho Nacional de Desenvolvimento Cientifico e Tecnologico $(\mathrm{CNPq})$ and Petroleo Brasileiro (PETROBRAS) for the financial support. Particular appreciations are extended to N. Azambuja (PETROBRAS), F. Chemale Jr. (UFRGS), C. H. Nowatski, T. Dutra, E. L. Lavina (UNISINOS), A. Martin (Emory University), N. J. Butterfield (University of Western Ontario) and the RBG reviwers who helped, in many different ways, the development of the research and improvement of the final version of the manuscript.

* UNISINOS - Mestrado em Geologia - Av. Unisinos, 950 - CEP 93020-000 - São Leopoldo - RS - Brazil- Phone: 55515903333 Ext. 1743 - Fax: 5551 5908177 Email: ppaim@dgeo.unisinos.tche.br

** Bolsista de Iniciação Científica CNPq - PIBIC/UNISINOS

*** Universidade Federal do Mato Grosso - Departamento de Recursos Minerais - Av. Fernando Correa s/n - Cuiaba - MT - Brazil - CEP $78060-900$ - Phone: 55 653158750 Fax: 55653158752 - Email: atila@cpd.ufmt.br 


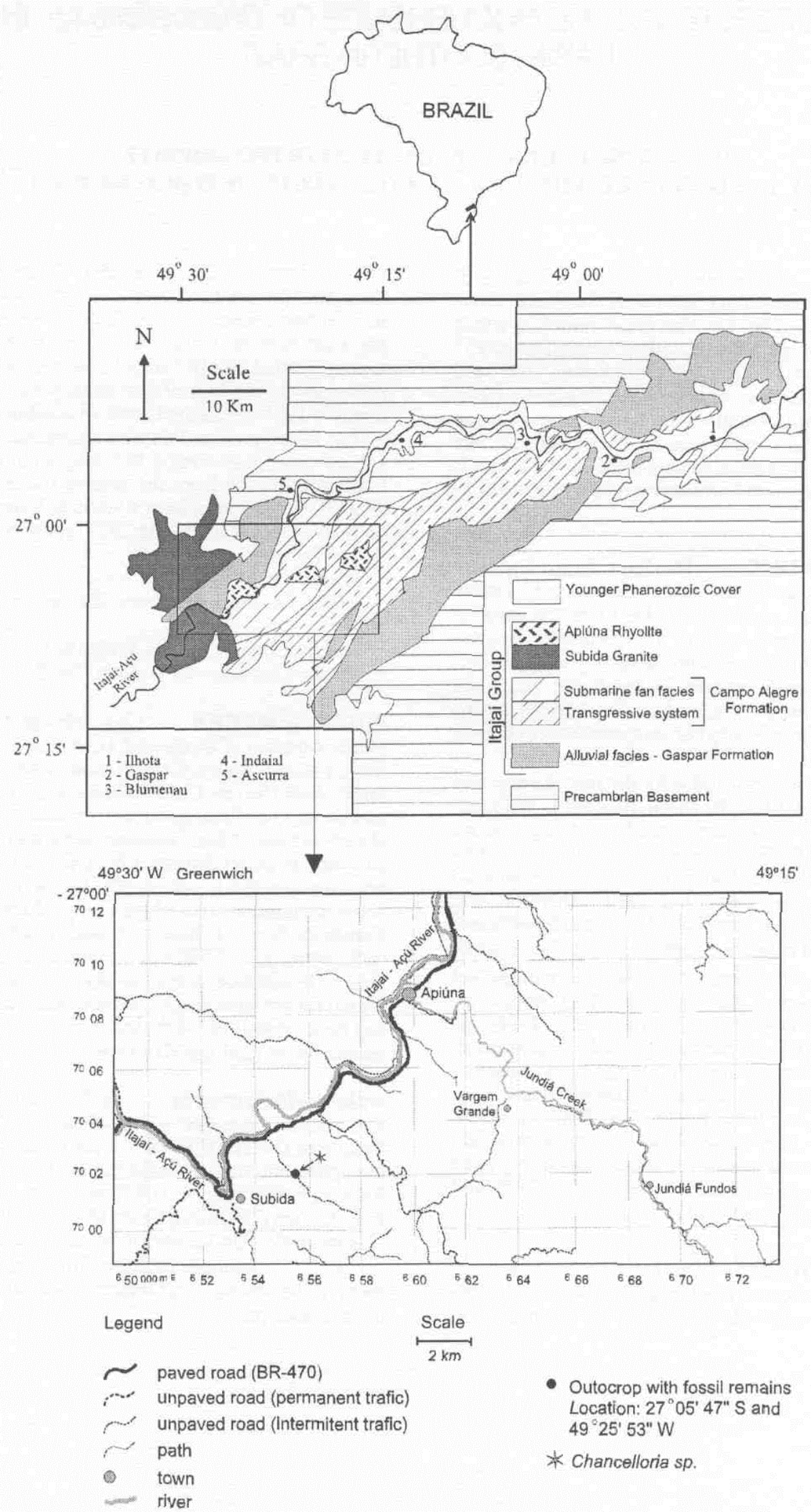

Figure 1 - Location and geological setting of the Itajai Basin (modified from Rostirolla 1991) and location of the road cut containing the fossils here described.

Figura 1 - Localização e contexto geológico da Bacia do Itajaí (modificado de Rostirolla 1991) e Iocalização do afloramento contendo os fósseis descritos neste artigo. 


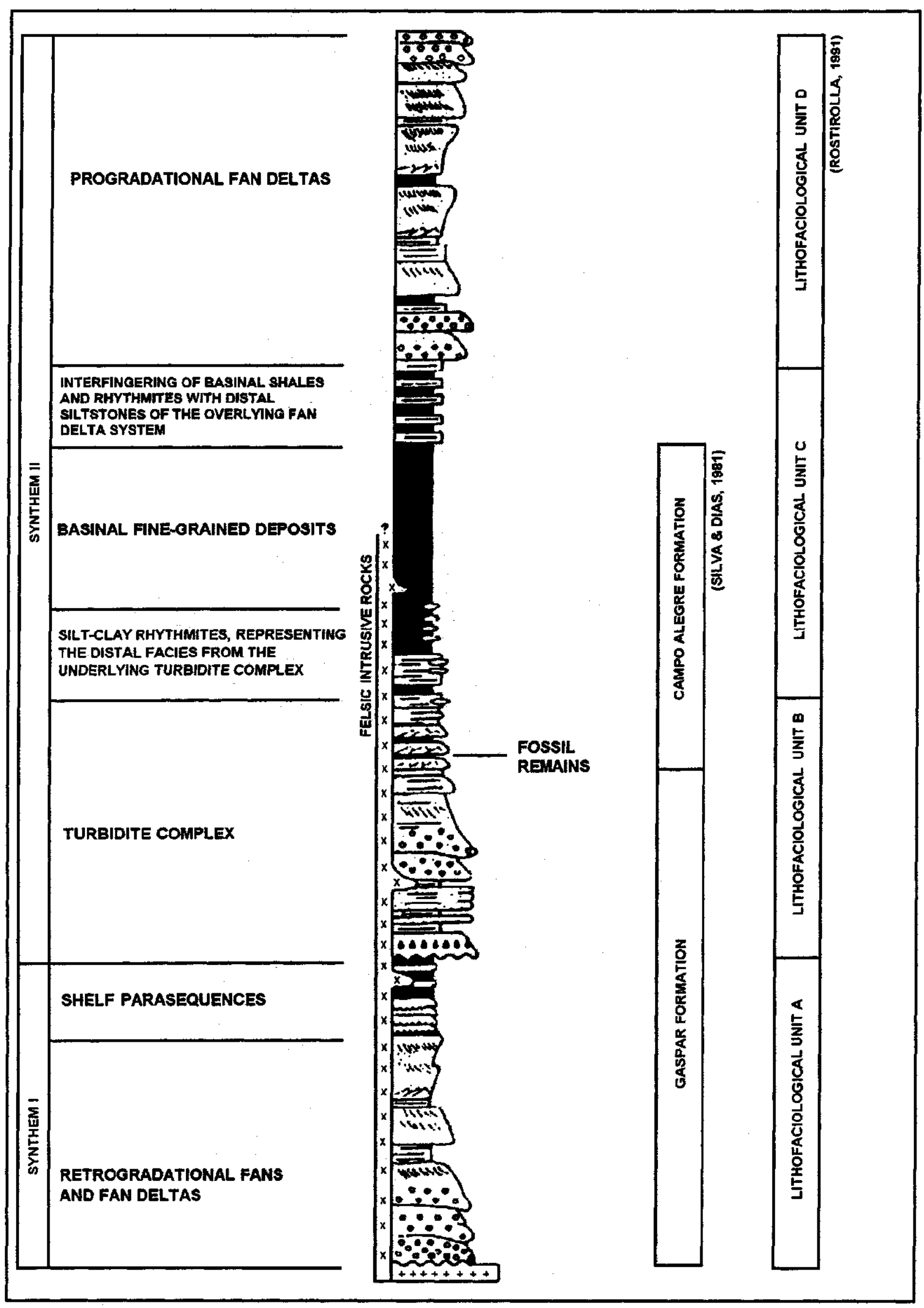

Figure 2 - Schematic geological column of the Itajai Basin (modified from Rostirotta \& Figueira 1995). Figura 2 - Coluna geoló gica esquemática da Bacia do Itajaí (modificado de RostiroIIa \& Figueira 1995). 


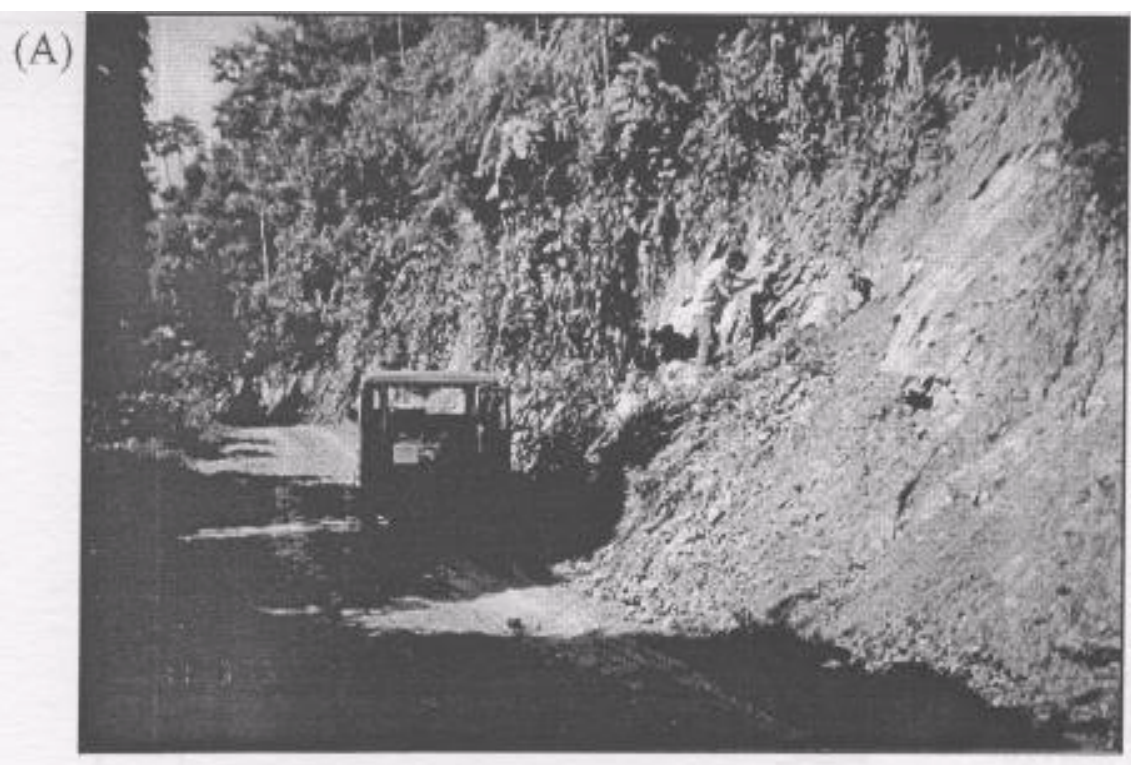

(B)
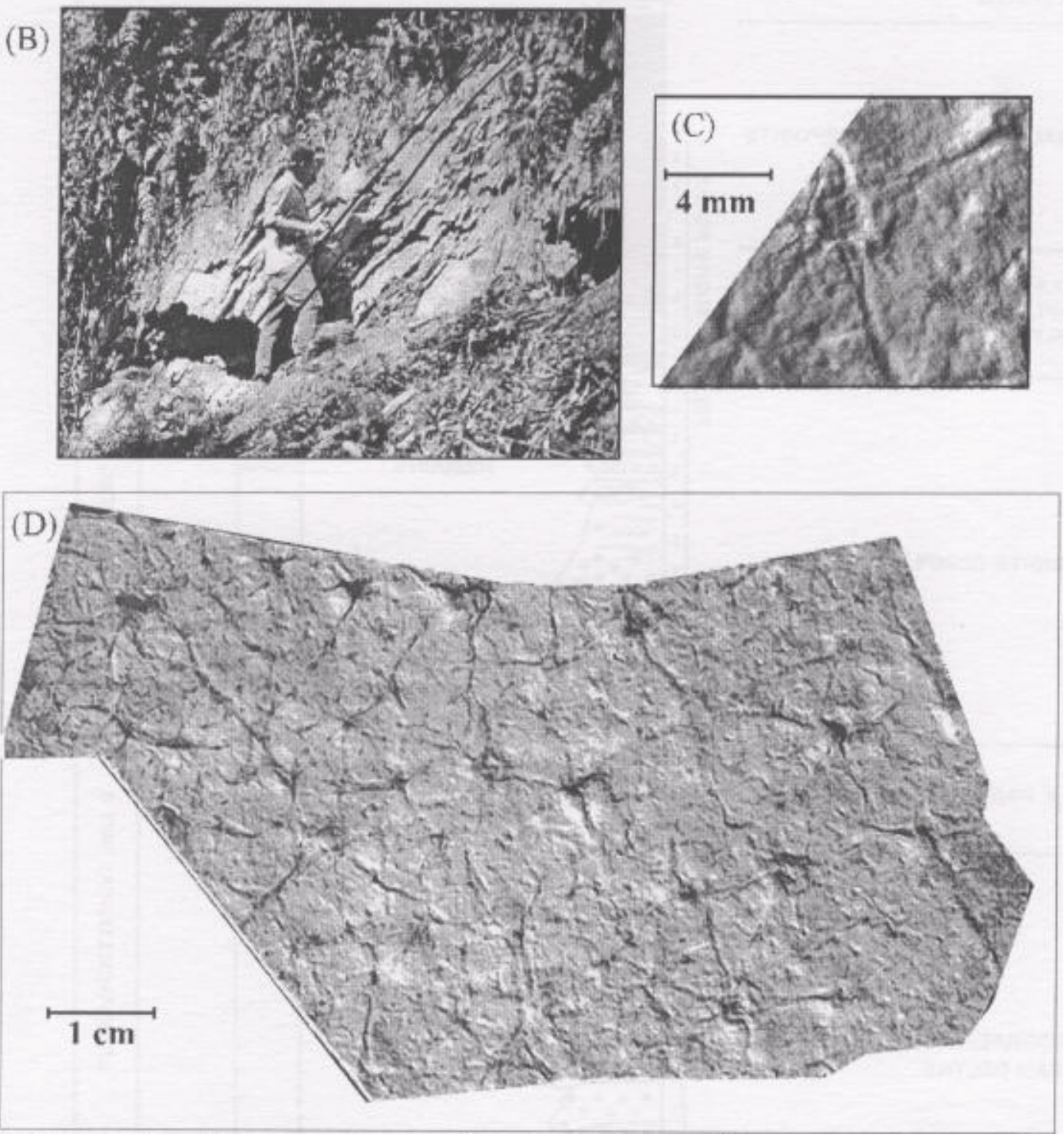

Figure 3 - (A) General view of the outcrop here described; (B) closer view of the outcrop indicating the fossiliferous horizon (between the black lines); (C) detailed view of a sclerite showing the central disk and the tangential rays thinning out from the central disk and presenting smoth, longitudinal depressions; and (D) general view of amalgamated and broken sclerites. Figura 3 - (A) Vista geral do afloramento descrito; (B) vista de detalhe do afloramento com a indicação do horizonte fossilffero (entre as linhas negras); (C) vista de detalhe de um esclerito mostrando o disco central e os bracos afinando a partir do disco central e apresentando suaves depressões longitudinais; e (D) vista geral dos escleritos amalgamados e parcialmente quebrados. 
Table 1 - Age and global distribution of Chancelloria sp.

Tabela 1 - Idade e distribuifao global da Chancelloria sp.

\begin{tabular}{|c|c|c|c|}
\hline Country & Locality and Geological Unit & Age & Author \\
\hline Canada & Mount Cap Fm - Mackenzie Mountains & early Middle Cambrian & Butterfield (1995) \\
\hline Canada & $\begin{array}{l}\text { Mount Cap Fm - Little Bear Shale - } \\
\text { Mackenzie Mountains }\end{array}$ & Lower to Middle Cambrian & \begin{tabular}{|l} 
Butterfield \& Nicholas \\
$(1996)$
\end{tabular} \\
\hline Canada & Burgess Shale - British Columbia & Cambrian & Morris (1992) \\
\hline USA & Wheeler Shale - Utah & Middle Cambrian & Gunther \& Gunther (1981) \\
\hline USA & Slade belt - New York & late Early Cambrian & $\begin{array}{l}\text { Landing \& Bartowski } \\
(1996)\end{array}$ \\
\hline USA & $\begin{array}{l}\text { Marjum Limestone - House Range and } \\
\text { Drum Mountains Utah }\end{array}$ & Lower Cambrian & Rigby (1983) \\
\hline Greenland & Paradisfjeld Group & early Middle Cambrian & Peel \& Higgins (1980) \\
\hline Great Britain & Comley Limestone & Cambrian & Reid (1959) \\
\hline France & $\begin{array}{l}\text { "Gres et Calcaires de Limousis" and } \\
\text { "Gres de Marcory" Montagne Noire }\end{array}$ & Lower Cambrian & Geyer (1986) \\
\hline Italy & $\begin{array}{l}\text { Cabitza Fm (Nodular Limestone } \\
\text { Member) - SW Sardinia }\end{array}$ & Middle Cambrian & $\begin{array}{l}\text { Cherchi \& Schroeder } \\
\text { (1984) }\end{array}$ \\
\hline Soviet Union & Siberia/Mongolia & \begin{tabular}{|l|} 
Lower Cambrian \\
Tommotian/Botomian \\
\end{tabular} & $\begin{array}{l}\text { Rozanov \& Zhuravlev } \\
\text { (1992) }\end{array}$ \\
\hline Soviet Union & Siberia & Tommotian & $\begin{array}{l}\text { Missarzhevsky \& } \\
\text { Rozanov (1981) }\end{array}$ \\
\hline Soviet Union & Lena River Section & Tommotian & $\begin{array}{l}\text { Matthews \& } \\
\text { Missarzhevsky (1975) }\end{array}$ \\
\hline China & Yangtze region & $\begin{array}{l}\text { Lower Cambrian } \\
\text { (Meishucunian) }\end{array}$ & Zhi-Wen (1992) \\
\hline China & & $\begin{array}{l}\text { Lower Cambrian } \\
\text { (Tommotian) }\end{array}$ & Pojeta \& Zhang (1983) \\
\hline $\begin{array}{l}\text { Spain and } \\
\text { North Africa }\end{array}$ & & Lower to Upper Cambrian & Sdzuy (1969) \\
\hline $\begin{array}{l}\text { Senegal and } \\
\text { Guinea }\end{array}$ & Taoudeni Basin West Africa & $\begin{array}{l}\text { Early/Middle(?) } \\
\text { Cambrian }\end{array}$ & Culver et. al. (1996) \\
\hline South Australia & & Early Cambrian & Bengtson et al. (1990) \\
\hline Australia & $\begin{array}{l}\text { Wirrealpa Limestone } \\
\text { (Flinders Ranges) }\end{array}$ & Cambrian (Toyonian) & Brock \& Cooper (1993) \\
\hline Argentine & $\begin{array}{l}\text { Formacion Empozada } \\
\text { (Mendoza) }\end{array}$ & Cambrian & Beresi \& Heredia (1995) \\
\hline Argentine & $\begin{array}{l}\text { La Laja Fm and San Isidro Fm - Chica } \\
\text { de Zonda Range/ Empozada Gulch }\end{array}$ & $\begin{array}{l}\text { upper Lower to Middle } \\
\text { Cambrian }\end{array}$ & Beresi \& Rigby (1994) \\
\hline
\end{tabular}

\section{REFERENCES}

Almeida, F. F. M. de. 1967. Origem e evolução da plataforma brasileira. Rio de Janeiro. DNPM/DGM, 36p (Boletim 241).

Appi, C. J.; Souza Cruz, C. E.; Barrocas, S. L. \& Freitas, E. L. 1987. Modelo deposicional dos turbiditos do Proterozóico Superior do Grupo Itajaí SC. Boletim do Núcleo de Minos Gerais da SBG, 6: 31-46.

Appi, C. J. \& Souza Cruz, C. E. 1990. Estratigrafia de Sequencias na Bacia do Itajaí. In: CONGRESSO BRASILEIRO DE GEOLOGIA, 36, Natal, 1990. Anais..., Natal, SBG, V.I, p.93-107.

Basei, M. A.; Kawashita, K. \& Siga Jr., O. 1987. Idade, características litoestratigráficas e estruturais do Grupo Itajaí, Santa Catarina. In: SIMF'SIO SUL-BRASILEIRO DE GEOLOGIA, 3, Curitiba, 1987. Atas..., Curitiba, SBG, V. 1, p. 93-106.

Bengtson, S.; Morris, S. C.; Cooper, B. J.; Jell, P. A. \& Runnegar, B. N. 1990. Early Cambrian fossils from South Australia. Assoc. of Australasian Palaeontologists, Memoir 9: 45-47.
Bengtson, S. \& Missarzhervski, V. V. 1981. Coeloscleritophora - a major group of enigmatic Cambrian metazoans. United States Geological Survey Open File Report, 81-743: 19-21.

Beresi, M. S. \& Rigby, J. K. 1994. Sponges and Chancelloriids from the Cambrian of Western Argentina. J. Paleont., 68 (2): 208-217.

Beresi, M. S. \& Heredia, S. 1995. Associacion de espiculas de poriferos cambricos de la Formacion Empozada, Precordillera de Mendoza. Ameghiniana (Rev. Assoc. Paleont. Argent.), 32 (4): 401-405.

Brock, G. A. \& Cooper, B. J. 1993. Shelly fossils from the Early Cambrian (Toyonian) Wirrealpa, Aroona Creek, and Ramsay limestones of south Australia. J. Paleont., 67 (5): 758-787.

Butterfield, N. J. \& Nicholas, C. J. 1996. Burgess Shale-type preservation of both non-mineralizing and "shelly" Cambrian organisms from the Mackenzie Mountains, northwestern Canada. J. Paleont., 70 (6): 893-899. 
Cherchi, A. \& Schroeder, R, 1984, Middle Cambrian foraminifera and other microfossils from SW Sardinia. Bollettino delta Societd Paleontologica Italiana, 23(2): 149-160.

Ciguel, J. H. G.; Góis, J. R. \& Acefiolaza, F. G, 1992 Ocorrência de icnofosseis em depd-sitos mola'ssicos da Formação Camarinha (Neoproterozóico III - Cambriano Inferior), no Estado do Paraná Brasil. Universidad Nacional de Tucuman, Série Correlation Geológica, 9. 157-158.

Culver, S. J.; Repetsky, J. E.; Pojeta, J. \& Hunt, D. 1996. Early and Middle (?) Cambrian Metazoa and Protistan fossils from west Africa. $J$. Paleont., 70 (1): 1-6.

Geyer, M. 1986. Découverte de microfaune dans le Cambrien infériur du versant meridional de la Montagne Noire (Cabardes et Minervois, Aude - France), Bull Soc. Hist. Nat. Toulouse, 122: 11-17.

Gresse, P. G.; Chemale Jr., F.; da Silva, L. C; Walraven, F. \& Hartmann, L. A. 1996. Late- to post-orogenic basins of the Pan-African-Brasiliano collision orogen in southern Africa and Southern Brazil. Basin Research, 8: 157-171.

Gunther, L. F. \& Gunther, V. G. 1981 Some Middle Cambrian fossils of Utah. Geology Studies, Brigham Young University, $81 \mathrm{pp}$

Krebs, A. S. J.; Lopes, R. C. \& Cammozato, E. 1990. Caracterizacão faciológica do Grupo Itajaf na Folha Botuverá (SC). In: CONGRESSO BRASILEIRO DE GEOLOGIA, 36. Natal, 1990. Anais..., V. 1, p. 82-92.

Landing, E. \& Bartowski, K. E. 1996. Oldest shelly fossils from the laconic allochthon and late Early Cambrian sea-levels in eastern Laurentia, $J$. Paleont, 70 (5): 741-761.

Leipnitz, I.I. ; Paim, P.S.G.; Da Rosa, A.A.S.; Zucatti da Rosa, A.L. \& Nowatski, C. H. 1997 Primeira occorencia de Chancelloridae no Brasil. In: CONGRESSO BRASILEIRO DE PALEONTOLOGIA, 15, Sao Pedro, Agosto de 1997. Boletim de Resumos, UNESP, p. 01.

Macedo, M. H. F.; Basei, M. A, S.; Bonhomme, M. G. \& Kawashita, K. 1984 Dados geocronológicos referentes as rochas metassedimentares do Grupo Itajaí (SC). Revista Brasileira de Geociencias, 14 (1): 30-34.

Matthews \& Missarzhevsky, V. V. 1975 Small shelly fossils of Late Precambrian and early Cambrian age: a review of Recent work. Quarterly Journal of the Geological society of London, 131: 289-304,

Missarzhevsky, V. V, \& Rozanov, A. Y. 1981 Fossil assemblages and zonal stratigraphy of Cambrian-precambrian boundary deposits of the Siberian Platform. In: RAABEN, M. E. ed. The Tommotian Stage and the Cambrian Lower Boundary Problem, p. 62-70.

Mora, S. 1993. Stratigrafta fisica e analisi di fades del complesso torbiditico Itajai (Proterozoico Superiore). Rio de Janeiro, 18p (Relazione de Tirocino -PETROBRAS)

Morris, S. C. 1992, Burgess Shale-type faunas in the context of the "Cambrian explosion": a review. Journal of the Geological Society, 149:631 -636.
Netto, R. G.; Paim, P. S. G. \& Da Rosa, C. L. M. 1992. Informe preliminar sobre a ocorrencia de tragos fósseis nos sedimentitos das bacias do Camagua e Santa Barbara. In: WORKSHOP SOBRE AS BACIAS MOLASSICAS BRASILIANAS, 1, São Leopoldo, 1992. Resumos Expandidos, UNISINOS, p. 90-96.

Netto, R. G. \& Zucatti da Rosa, A.L. 1997. Registro icnofossilífero da Bacia do Itajaf, SC: uma primeira visao. In: CONGRESSO BRASILEIRO DE PALEONTOLOGIA, 15, Sao Pedro, Agosto de 1997. Boletim de Resumos, UNESP, p. 136.

Nicholas, N. J. 1995. Chancelloriids from the Middle Cambrian of the Mackenzie Mountains: inplications for early poriferan evolution. Geological Society of America, Annual Meeting, New Orleans, USA, A-269.

Peel, J. S. \& Higgins, A. K. 1980. Fossils from the Paradisfjeld Group, North Greenland fold belt. Rapp. Grmlands geol. Unders, 101: 28 .

Pojeta, J. \& Zhang, R. (1983) The oldest shelly faunas. Abstracts with programs, The British Library (ref. 31549), p. 662.

Reid, R. E. H. 1959. Occurrence of Chancelloria Walcott in the Comley Limestone. Geological Magazine. 96(3): 261-262.

Rigby, J. K. 1983. Sponges of the Middle Cambrian Marjum Limestone from the House Range And Drum Mountains of Western Millard County, Utah Journal of Paleontology, 57: 240-270.

Rostirolla, S. P. 1991. Tectônica e sedimentacdo da Bacia do Itajaí - SC. Ouro Preto, 131p. (Dissertação de Mestrado, Universidade Federal de Ouro Preto).

Rostirolla, S. P. \& Figueira, E. G. 1995. Associagoes de fades turbidfticas do Grupo Itajaf, SC: sedimentag5o de agua profunda em uma bacia de antepafs. Boletim Paranaense de Geociencias, 43: 79-94.

Rozanov, A. Y. \& Zhuravlev, A. Y. 1992. The Lower Cambrian fossil record of the Soviet Union. In: Lipps, J. H. \& Signor, P. W., eds. Origin and Early Evolution of the Metazoa. New York, Plenum Press, p. 205-283.

Sdzuy, K. 1969. Unter und Mittelkambrische Porifera (Chancelloriida und Hexactinellida). Palaont. Z. 43 (3/4): 115-147.

Silva, L. C. da \& Dias, A. A. 1981. Projeto Timbo - Barra Velha, Brasil. Porto Alegre, 282p (Convenio DNPM/CPRM - relatono inedito).

Walcott, C.D.I 920. Middle Cambrian Spongiae. Smithsonian Miscellaneous Collections, 67: 261-364.

Zhi-Wen, J. 1992. The Lower Cambrian fossil record of China. In: Lipps, J. H. \& Signor, P. W., eds. Origin and Early Evolution of the Metazoa. New York, Plenum Press, p. 311-333.

MANUSCRITO NB-14

Recebido em 30 de agosto de 1997

Revisao dos autores em 03 de novembro de 1997 Revisao aceita em 04 de novembro de 1997 